\title{
COMPOSITION DES COMMISSIONS
}

(Arrêtée en séance pleniere à Stockholm, le ro aont, 1938)

3. Commission des Notations, des Unités, et de l'Économie des Publications.

Président: M. E. Strömgren.

Membres: MM. Bosler, Chant, Eddington, L. Grabowski, Horn d'Arturo Lou Kao, Krassowski, Russell, Schlesinger, Slouka, B. Strömgren.

4. Commission des Éphémérides.

Président: M. Fayet.

Membres: MM. Carnera, Comrie, Fukumi, Herrero, Michkovitch, Robertson, Sadler.

5. Commission des Analyses de Travaux et de Bibliographie.

Président: M. Lundmark.

Membres: MM. P. Bourgeois, Brasch, J. Chatelu, Collinder, Mme d'Azambuja, MM. Dittrich, Emanuelli, Gabba, E. Gastardi, Neubauer, Nordenmark, Norman, Sampson, Selga, Yaschnov.

6. Commission des Télégrammes astronomiques.

Président : M. Spencer Jones.

Directeur dU Bureau: M. E. Strömgren.

Membres: MM. B. H. Dawson, Shapley, Wood.

8. Commission de l'Astronomie méridienne.

Président D'Honneur: Sir F. W. Dyson.

Président: M. H. R. Morgan.

Membres: MM. Armellini, R. Baillaud, Boss, Carnera, Mme Chandon, MM. Cullen, Danjon, Dneprovsky, Fayet, Gyllenberg, Hasimoto, Hins, J. Jackson, Jeffers, Spencer Jones, A. Lambert, Moreau, Procházka, Rents, R. M. Stewart, van Herk, Zimmer.

Sous-commission pour la répétition du catalogue de 1'A.G.

Président: M. Schlesinger.

Membres: MM. Boss, J. Jackson, Spencer Jones, A. Lambert, Moreau, H. R. Morgan.

9. Commission des Instruments astronomiques.

Président D'Honneur: M. Ch. Fabry.

Président: M. Carroll.

Membres: MM. Anderson, R. Baillaud, Burch, Mme Chandon, MM. Chrétien, Couder, Danjon, C. R. Davidson, Dufay, Dunham, Hargreaves, Lallemand, Link, Lyot, McKellar, Minnaert, Moll, Ohman, H. H. Plaskett, J. S. Plaskett, Redman, Ross, Rosseland, Schilt, Svoboda.

10. Commission des Taches solaires et des Nombres caractéristiques.

PrÉsident: M. Brunner.

Membres: M. Abetti, Mlle Bloch, MM. Burton, S. Chapman, G. S. da Costa Lobo, d'Azambuja, Moss, Newall, Newton, Nicholson, Perepelkin, Pettit, Rodés, Rowland, Sekiguti, Sotome. 
11. Commission des Phénomènes chromosphériques.

President: M. d'Azambuja.

Membres: MM. Abetti, Brunner, Mme d'Azambuja, MM. Deslandres, Dobbie, Mlle Dodson, MM. dos Reis, Evershed, Lyot, McMath, Newbegin, Newton, Nicholson, Pettit, Richardson, Rodés, Rowland, Royds, Sotome, Waldmeier.

Sous-commission pour la Cinématographie des Protubérances.

PrÉsident: M. Lyot.

Membres: MM. d'Azambuja, N. Donitch, McMath, Waldmeier.

12. Commission de la Radiation solaire et de la Spectroscopie solaire.

Président: M. Abetti.

Membres: MM. Abbot, Babcock, P. Carrasco, Carroll, S. Chapman, d'Azambuja, Dingle, Evershed, A. S. King, Lyot, McCrea, Menzel, Milne, Minnaert, Mlle Nováková, MM. Pannekoek, Perepelkin, Pettit, H. H. Plaskett, Russell, Sekiguti, Shane, Mme C. E. Moore Sitterly, MM. Slouka, Waldmeier, Woolley, Yü.

18. Commission des Eclipses du Soleil.

PrÉsident: M. Mitchell.

Membres: MM. Abetti, Caŗroll, Curtís, F. M. da Costa Lobo, Danjon, C. R. Davidson, N. Donitch, Dunham, Freundlich, Gerasimovich, Horn d'Arturo, J. Jackson, Link, Lyot, Menzel, Minnaert, Sekiguti, Slouka, Stratton, Wood, Woolley, Yamamoto, Yü.

14. Commission des Etalons de Longueur d'Onde et des Tables de Spectres solaires.

PRESIDENT: M. Meggers.

Membres: MM. Babcock, Buisson, Burns, Edlén, Ch. Fabry, A. Fowler, Harrison, Nagaoka, Pérard, Sears.

15. Commission pour 1'Étude physique des Comètes.

Président: M. Baldet.

Membres: MM. Bobrovnikoff, P. Bourgeois, Crommelin, Dufay, S. V. Orlov, Quénisset, Swings, van Biesbroeck, Vorontsov-Velyaminov, Vsekhsvyatsky, Wilk.

16. Commission pour les Observations physiques des Planètes et des Satellites.

Président: M. Maggini.

Membres: MM. Antoniadi, S. Arend, Armellini, Mlle Blagg, MM. Campbell, Danjon, Delmotte, Delporte, Dunham, Hargreaves, Mlle Harwood, MM. Jarry-Desloges, Lampland, Link, Luplau-Janssen, Lyot, Nicholson, Peek, Phillips, Plakidis, Quénisset, F. E. Ross, E. C. Slipher, Ueta, F. E. Wright, W. H. Wright.

17. Commission de la Lune.

Président: M. Banachiewicz.

Membres: MM. Brouwer, Comrie, Danjon, Matukuma, Meyer, Sadler, E. Strömgren, Volta, Yakovkin. 


\section{Sous-commission des Occultations.}

Président: M. Brouwer.

Membres: MM. Banachiewicz, Comrie, Danjon, B. H. Dawson, J. Jackson, Spencer Jones, Meyer, Sadler, E. Strömgren.

18. Commission des Longitudes par Télégraphie sans Fil.

Président: Général G. Perrier.

Membres: MM. R. Baillaud, Bianchi, W. Bowie, de Lemos, Demetrescu, Mme Dubois, MM. Esclangon, Fayet, Guyot, de Graaff Hunter, Ivanov, Jeffers, Spencer Jones, Jouaust, Krassowski, A. Lambert, Lejay, Madwar, Michkovitch, Moreau, Niethammer, Nörlund, Pavlov, Peisino, Plakidis, Sekiguti, Silva, Sollenberger, R. M. Stewart, Stoyko, Svoboda, Ping-Jan Tsiang, Watts, Witkowski, Zimmer.

19. Commission de la Variation des Latitudes.

Président D'Honneur: M. Kimura.

Président: M. Spencer Jones.

Directeur du Bureau: M. Carnera.

Membres: MM. G. Bemporad, Berg, Bianchi, H. E. Burton, H. Castro, Esclangon, Hasimoto, W. D. Lambert, Michkovitch, Moreau, Niethammer, A. J. Orlov, Peres, Schlesinger, Sollenberger, Stetson, Stoyko, M. F. Subbotin, Svoboda, Vocca, E. L. Williams.

20. Commission des Positions et des Mouvements des petites Planètes, des Comètes, et des Satellites.

PrÉsident D'HonneUR: M. Leuschner.

Président: M. Delporte.

Vice-Président: M. Brouwer.

Membres: MM. S. Arend, Asplind, Banachiewicz, R. Carrasco, Comrie, Crawford, Crommelin, de la Villemarqué, Eckert, Esclangon, Fayet, Guth, Hammond, Heinrich, Herget, Hinks, K. Hirayama, Spencer Jones, Kamieński, Kepiński, Komendantov, Mme Laugier, MM. E. G. Martin, E. L. Martin, Mauderli, Maxwell, Michkovitch, Nechvíle, G. Neuymin, Perrine, Schaumasse, G. A. Shayn, Silva, Slocum, E. Strömgren, van Biesbroeck, Mlle Vinter Hansen, MM. Volta, Whipple, Wood.

Sous-commission de la Parallaxe solaire.

Président: M. Spencer Jones.

Membres: MM. Esclangon, Hammond, Hinks, E. G. Martin, Nechvíle, Perrine, Slocum.

Sous-commission pour 1'Etude des Comètes périodiques.

Président: M. Crommelin.

Membres: MM. Fayet, Kepiński, Mlle Vinter Hansen. 
22. Commission des Etoiles filantes, de la Lumière zodiacale et des Problèmes analogues.

President: M. de Roy.

Membres: MM. Astapovich, Boothroyd, Bosler, Cabannes, Cap, Chant, Dauvillier, M. Davidson, Dobson, N. Donitch, Dufay, Elvey, Fedynsky, Mme Flammarion, MM. Gatterer, Gauzit, Grouiller, Guth, Harang, Housman, Leonard, Link, Loreta, Millman, Nielsen, Olivier, Prentice, Rudaux, V. M. Slipher, Störmer, Svoboda, Vandekerkhove, Vegard, Watson, Yamamoto.

Sous-commission de la Lumière zodiacale.

Président: M. Yamamoto.

Membres: MM. Cap, N. Donitch, Elvey, Fesenkov, Housman, Loreta, V. M. Slipher.

Sous-commission de la Lumière du Ciel nocturne.

President: M. Dufay.

Membres: MM. Cabannes, S. Chapman, Dauvillier, Dufay, Elvey, Ch. Fabry, Fesenkov, Gauzit, Harang, Link, V. M. Slipher, Störmer, Struve, Vegard.

28. Commission de la Carte du Ciel.

Président d'Honneur: M. Esclangon.

Président: M. J. Baillaud.

Membres: MM. A. Bemporad, Bok, Bratu, Delporte, Delvosal, Dyson, Favaro, Gallo, Greaves, Herrero, Hertzsprung, J. Jackson, Spencer Jones, Knox-Shaw, Lagarde, Lindblad, Luyten, Meyer, Nissen, Oort, Paloque, L. Picart, H. H. Plaskett, Rougier, Russell, Schlesinger, Smart, Stein, van Rhijn, Warzée.

24. Commission des Parallaxes stellaires et des Mourements propres.

President D'Honneur: M. Schlesinger.

PRÉsidenT: M. J. Jackson.

Membres: MM. W. S. Adams, Alden, Asklöf, Bianchi, Cecchini, Finsen, Harper, Hertzsprung, A. Hunter, Jordan, Lindblad, Luyten, Mitchell, Nechvile, Russell, Shapley, Slocum, Smart, van de Kamp, van Maanen, van Rhijn, Voûte.

25. Commission de Photométrie stellaire.

PRÉsIdent: M. Hertzsprung.

Membres: MM. Atkinson, J. Baillaud, Belyawsky, A. Bemporad, Bennett, Bergstrand, Danjon, de Sitter, Dufay, Fesenkov, Mme Payne Gaposchkin, MM. Greaves, W. K. Green, J. S. Hall, Holm, Lindblad, Maggini, Malmquist, Oosterhoff, F. E. Ross, Rougier, Rybka, Schilt, Seares, Sekiguti, Shapley, Stebbins, Sternberk, Stoy, Tremblot, Vanderlinden, van Rhijn, von Zeipel.

Sous-commission des Etalons de Grandeur stellaire.

Président: M. Seares.

Membres: MM. W. K. Green, Hertzsprung, Lindblad, Shapley. 
Sous-commission des Séquences de Magnitudes.

Président: Mme Payne Gaposchkin.

MEMBREs: MM. Vyssotsky, Wallenquist.

26. Commission des Etoiles doubles.

Président: M. van den Bos.

Membres: MM. Aitken, Baize, Mme Bonnet, MM. B. H. Dawson, Finsen, Hertzsprung, Kuiper, Luyten, Rossiter, Russell, Strand, van Biesbroeck, Voûte, Woolley, Zagar.

27. Commission des Etoiles variables.

President: M. Dugan.

VICE-President: M. Grouiller.

Membres: MM. Banachiewicz, Campbell, Cox, Danjon, de Roy, Einbu, Gadomski, Gaposchkin, Gerasimovich, Mlle Harwood, MM. Hertzsprung, Jacchia, Jordan, Joy, Kopal, Kordylewski, Kukarkin, Lacchini, Lindley, McLaughlin, W. Chr. Martin, Merrill, Mitchell, A. V. Nielsen, Pearce, Pickering, Ryves, Shapley, Silva, Steavenson, Stebbins, Stein, E. Strömgren, Tiercy, Tsesewich, van Gent, Voûte, Wesselink.

28. Commission des Nébuleuses et des Amas stellaires.

Président: M. Shapley.

Membres: MM. Ambartsumian, Bowen, Carpenter, Cuffey, de Kerolyr, Henyey, Mme H. B. Hogg, MM. Hubble, Humason, Lampland, Lemaître, Lindblad, Lundmark, McVittie, Madwar, Mineur, Mlle J. Mohr, MM. Paraskévopoulos, Parvulesco, Reynolds, V. M. Slipher, Struve, Trumpler, Vanderlinden, van Maanen, Vyssotsky, Wallenquist, W. H. Wright, Zanstra.

29. Commission des Spectres stellaires.

Président: M. Struve.

Membres: MM. W. S. Adams, Beals, Mlle Cannon, MM. Cecchini, Chalonge, Colacevich, Dunham, Edwards, Mme Payne Gaposchkin, MM. Gerasimovich, F. S. Hogg, Joy, Lindblad, McLaughlin, Merrill, Milne, Minkowski, W. W. Morgan, H. H. Plaskett, J. S. Plaskett, Redman, Russell, Stratton, Swings, Thackeray, E. G. Williams, W. H. Wright.

Sous-commission des Novae.

PRÉsident: M. Stratton.

Membres: M. Cecchini, Mme Payne Gaposchkin, MM. McLaughlin, Merrill, Minkowski, W. H. Wright.

30. Commission des Vitesses radiales stellaires.

Président: M. W. S. Adams.

Membres: MM. Bok, Harper, F. S. Hogg, Joy, McLaughlin, Merrill, J. H. Moore, Neubauer, Pearce, J. S. Plaskett, Redman, Sanford, G. A. Shayn, Struve, R. K. Young. 
Sous-commission des Vitesses radiales fondamentales.

Président: M. Sanford.

Membres: MM. J. H. Moore, Pearce.

Sous-commission de Coordination des Observations des Vitesses radiales.

President: M. J. H. Moore.

Membres: MM. Harper, Joy, McLaughlin, G. A. Shayn, Struve.

Sous-commission des Longueurs d'Onde.

PrEsident: M. Harper.

Membres: MM. Merrill, Struve.

31. Commission de 1'Heure.

PrÉsident: M. Nörlund.

Vice-Président: Général Perrier.

Directeur DU BureaU: M. Esclangon.

Membres: MM. R. Baillaud, Banachiewicz, Bianchi, Carnera, Dneprovsky, Fayet, Gallo, Guyot, Hellweg, A. Lambert, Lejay, Moreau, Niethammer, L. Picart, Postoev, Preipich, Sampson, Sekiguti, Silva, H. Smith, Sollenberger, R. M. Stewart, Stoyko, Tinoco, Verbaandert, Voûte.

32. Commission des "Selected Areas".

Président: M. van Rhijn.

Membres: MM. W. S. Adams, Alden, E. A. Baker, Mme Payne Gaposchkin, MM. Hins, Knox-Shaw, Oort, Schlesinger, Seares, Shapley, van Maanen, R. K. Young.

83. Commission de la Statistique stellaire.

Président: M. Lindblad.

Membres: MM. Bok, Dziewulski, Eddington, Freundlich, Gratton, Gyllenberg, Hertzsprung, S. Hirayama, Lundmark, Luyten, Malmquist, Mineur, J. M. Mohr, Nordström, Ogorodnikov, Oort, Pannekoek, Parvulesco, Russell, Schalén, Schilt, Seares, Shapley, Stenquist, Strömberg, Trumpler, van de Kamp, Vanderlinden, van Hoof, van Maanen, van Rhijn.

84. Commission de la Matière interstellaire.

Président: M. Stebbins.

Membres: MM. Beals, Bertaud, Dufay, Dunham, Eddington, Greenstein, J. S. Hall, Merrill, Minkowski, J. S. Plaskett, Schalén, Struve, Swings, Trumpler, Whitford, E. G. Williams, O. C. Wilson.

85. Commission de la Constitution des Etoiles.

Président: M. Russell.

Membres: MM. Atkinson, Cowling, Eddington, Gamow, Hagihara, Jeans, Kopal, Milne, Pannekoek, Rosseland, B. Strömgren, Tiercy, von Zeipel, Woltjer. 
36. Commission de la Spectrophotométrie.

Président: M. Minnaert.

Membres: MM. Atkinson, Barbier, Beals, Carroll, Chalonge, Dufay, Dunham, Eddington, Elvey, Ch. Fabry, Greaves, F. S. Hogg, A. S. King, Lindblad, Menzel, Milne, Mulders, Ohman, Page, Pannekoek, H. H. Plaskett, Redman, Righini, Rosseland, Russell, Struve, Thackeray, Whipple, E. G. Williams, Woolley, Yü.

\section{COMMISSIONS MIXTES}

(Membres additionnels de Commissions mixtes élus par l'Union Internationale de Géodésie et Géophysique.)

18. Commission des Longitudes par Télégraphie sans Fil.

SEcrétaIre: M. Seligmann.

Membres: MM. Baeschlin, Banachiewicz, Beneł̌, Boulnois, Carnera, F. M. da Costa Lobo, Dodwell, Ferri, Garner, Gastardi, Giotti, S. Hirayama, Jelstrup, Jolly, Kennelly, E. Kohlschütter, Magliocco, Mailles, Nusl, Ogilvie, Romagna-Manoia, Rosén, Sotome, Tanakadate, Tinoco, Warchalowski, le Chef de l'Institut géographique, Direction de Géographie, Météorologie et Hydrologie, Mexique.

19. Commission de la Variation des Latitudes.

Membres: MM. Bowie, F. M. da Costa Lobo, Jelstrup, Nušl, Rosén, Schlesinger, Swick, Tanakadate.

81. Commission de 1'Heure.

Membres: MM. Bowie, Clough, Hinks, Jelstrup, Jolly, Spencer Jones, Jouaust, Loomis, Ogilvie, Somville. 